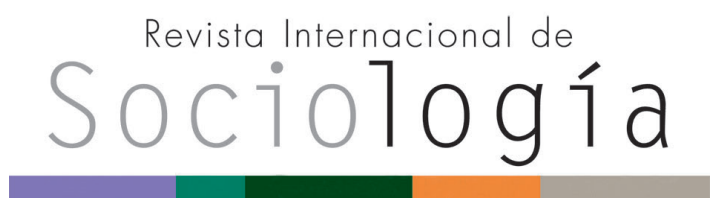

Revista Internacional de Sociología RIS vol. 74 (3), e042, julio-septiembre, 2016, ISSN-L:0034-9712 doi: http://dx.doi.org/10.3989/ris.2016.74.3.042

\section{VENDIENDO SOLUCIONES. Campañas tradicionales y profesionalizadas en Chile}

\author{
Maite de Cea \\ Universidad Diego Portales, Santiago de Chile. \\ maite.decea@udp.cl \\ Claudio Fuentes SaAvedra \\ Universidad Diego Portales, Santiago de Chile. \\ claudio.fuentessaa@udp.cl
}

Como citar este artículo / Citation: De Cea, M. y C. Fuentes S. 2016. "Vendiendo soluciones. Campañas tradicionales y profesionalizadas en Chile". Revista Internacional de Sociología 74(3):e042. doi: http://dx.doi.org/10.3989/ ris.2016.74.3.042

\section{SELLING SOLUTIONS. Traditional and professionalized campaigns in Chile}

Copyright: ( 2016 CSIC. Este es un artículo de acceso abierto distribuido bajo los términos de la licencia Creative Commons Attribution (CC BY) España 3.0.

Recibido: 29/10/2014. Aceptado: 18/09/2015.

Publicado on line: 22/08/2016

\section{Abstract}

This paper studies the relationship between candidates and the electorate through a in-depth analysis of three political campaigns for the Chamber of deputies in Chile in 2013. Confirming recent findings, this article shows the dominance of non-programmatic or clientelistic linkages between the electorate and representatives which implies the transaction of votes for information, material resources and influence. The paper distinguishes two campaign strategies: a traditional strategy supported on historical patterns of intermediation, and a professionalized one which rationalize and makes an efficient allocation of resources. The study makes a relevant empirical as well as conceptual contribution to the study of the patterns of political intermediation in social contexts of social disaffection.

\section{KeYWORDS}

Clientelism; Elections; Indigenous Peoples; Political Campaigns; Political Parties.

\author{
Campañas políticas; Clientelismo; Elecciones; Partidos \\ políticos; Pueblos indígenas.
}




\section{INTRODUCCIÓN}

En comparación con la mayoría de América Latina, a Chile se le ha caracterizado como un país altamente institucionalizado y estable, con partidos relativamente longevos y con cierto nivel de coherencia programática entre representantes y electores (Mainwaring y Scully 1995; Valenzuela 1995; Tironi y Agüero 1999). Siguiendo la literatura, los sistemas democráticos con estas características tendrían menos incentivos para utilizar prácticas de tipo clientelar (Levitsky y Murillo 2005).

Sin embargo, otros autores han desafiado estos argumentos, mostrando evidencia sistemática de la presencia de patrones de vínculo no-programático en la búsqueda de votos por parte de candidatos al Congreso Nacional (Barozet 2003; Díaz et al. 2006; Arriagada 2013). Algunos sostienen que ciertos partidos tendrían la capacidad de segmentar sus estrategias social y territorialmente, lo que explicaría por ejemplo el éxito de partidos que a nivel nacional tienen un discurso alejado de sus electores (Luna 2010).

Nuestro trabajo evaluará hasta qué punto predominan las estrategias programáticas vs. clientelares en campañas políticas a partir de un estudio en profundidad de tres campañas desarrolladas en el sur de Chile. Se trata de un estudio inédito que combina entrevistas y etnografía de campaña ganando en riqueza descriptiva. Esto nos permite entregar importantes pistas sobre el desarrollo de patrones de relación partidos-electores en un contexto político de creciente desafección política.

Como nos interesaba indagar en la relevancia de los aspectos programáticos/no programáticos, seleccionamos un territorio que cumpliera con dos condiciones esenciales para testear nuestra argumentación: distritos con altos niveles de pobreza y alta presencia de población indígena. La primera condición usualmente es considerada por la literatura como un incentivo para la generación de prácticas clientelares. La presencia indígena es, en cambio, una condición relevante para dividir a los candidatos desde el punto de vista programático. Mientras a nivel nacional los partidos de centro-izquierda han insistido en políticas de reconocimiento indígena, los partidos de la derecha han enfatizado políticas de inclusión productiva para resolver sus demandas.

El artículo resulta relevante por tres razones: a) buscamos expandir el foco de interés desde los grandes centros urbanos hacia zonas más periféricas de modo de evaluar hasta qué punto los patrones de vínculo representante-electores difieren (Durston 2009); b) quisimos explorar particularmente el tipo de vínculo que se desarrolla entre candidatos y electores en un tema altamente programático a nivel nacional, que ha sido muy controvertido en la región de la Araucanía y que constituye hoy uno de los principales focos de conflictividad social del país; y c) buscamos aportar a la literatura que explora la evolución de los partidos políticos en sus grados de institucionalización (o desinstitucionalización). Al estudiar cómo los partidos despliegan sus campañas concretamente observaremos el peso del debate ideológico y el rol que cumplen las estructuras de partidos en la obtención de votos.

\section{Marco teórico}

Nuestro estudio se enmarca en lo que la literatura ha venido discutiendo sobre las transformaciones y/o continuidades en las relaciones partidos-electores en democracias emergentes. A diferencia de otros países latinoamericanos, Chile aparece como un caso con dos características distintivas: organización partidaria programática por clivajes sociales y alto nivel de institucionalización de las entidades políticas (Mainwaring y Scully 1993; Siavelis 2000). No obstante, ambos supuestos han sido problematizados por diversos autores. Si bien se advierte que comparativamente todavía existen altos niveles de identificación programática tanto de los candidatos como de los electores (Calvo \& Murillo 2012), estudios específicos sobre el funcionamiento de campañas destacan la relevancia de los mecanismos de intermediación y el clientelismo para la obtención de votos. Mientras a nivel agregado nacional se observa una fuerte continuidad en partidos y clivajes ideológicos, cuando se examinan las prácticas políticas cotidianas se advierte mucha mayor precariedad institucional y mayores niveles de vinculaciones no programáticas (Rehren 1996; Espinoza 2006; Durston 2009; Luna 2010; Contreras 2012; Arriagada 2013).

Esta alta institucionalidad de los partidos también ha sido cuestionada y se expresa en una fuerte caída en la confianza social en los partidos e instituciones representativas, baja cercanía con los partidos, pérdida de adhesión ciudadana al eje izquierdaderecha, y fuerte caída en la participación electoral (Fuentes et al. 2006; Altman 2006; Luna y Rosenblatt 2012, entre otros). Así, la aparente estabilidad del sistema de partidos no estaría dada tanto por los vínculos verticales partidos/sociedad sino que por otras condiciones estructurantes del sistema como ciertas reglas que definen el juego político (Constitución, ley electoral, ley de partidos), y las condiciones socioeconómicas que podrían facilitar/obstaculizar la acción colectiva a nivel político.

Las tendencias antes descritas (estabilidad y división en clivaje de partidos a nivel macro y vínculos no programáticos a nivel micro) podrían ser tendencias no contradictorias. Luna (2010) al analizar el caso del éxito de la UDI -el partido más conservador de Chile- sostiene que dicho partido ha desarrollado una estrategia dual de representación que apela simultáneamente a los sectores ricos de la población (que le aportan apoyo político y dinero) y a los sectores pobres donde tiene una alta penetración. La exis- 
tencia de una poderosa estructura organizacional de base le permitió desarrollar vínculos no programáticos con los sectores populares del país. A ello se sumó un liderazgo presidencial de un candidato de la UDI (Joaquín Lavín) que casi obtuvo la presidencia y que ayudó a potenciar el partido.

Luna advierte que este patrón es común en América Latina. En contextos de alta fragmentación social, la competencia política de los partidos se da a partir de una estrategia de segmentación de los electorados en los distritos en que se compite, lo que es verticalmente integrado con una estrategia de posicionamiento nacional (Luna 2012: 329-330). El vínculo no programático se daría a partir de las donaciones privadas que permiten aceitar vínculos clientelares; o de la provisión de acceso privilegiado a recursos del Estado (pork barrel). Se entiende por clientelismo "el intercambio de bienes y servicios por apoyo político y votos" y que implica asimetría social entre los socios, reciprocidad y dependencia mutua, la personalización e informalización de los vínculos, y su carácter voluntario (Schroter 2010).

Consideramos que algunas de las condiciones señaladas por Luna en su trabajo sobre un partido se podrían extrapolar a otros partidos. Primero, el desarrollo de redes de intermediación local podría hacerse centralizada o descentralizadamente, a partir del trabajo individual de candidatos particulares. Segundo, la existencia de un liderazgo presidenciable nacional parece ser condición necesaria aunque no suficiente para determinar una elección a nivel senatorial o de diputados. En los casos que analizaremos advertimos que el vínculo entre el candidato y sus electores no pasaba por quién fuese la figura presidencial. De hecho, uno de los candidatos que seguimos tuvo el crecimiento electoral más importante de la zona pese a que la candidata presidencial de su sector sufrió la peor derrota desde 1993.

La literatura reciente que ha examinado la relación candidatos-electores de micro-nivel (distritos por ejemplo), señala la relevancia de los vínculos no programáticos a la hora de conquistar el poder. Szwarcberg (2013) estudia el caso argentino a nivel municipal y postula que además de la capacidad de utilizar prácticas clientelares, los candidatos deben preferir usarlas con el objetivo de movilizar a los votantes. Así, denomina a los candidatos 'pragmáticos' o 'idealistas', siendo los primeros quienes además de tener las capacidades, eligen tener una relación no programática con sus electores, mientras los segundos aunque son capaces de usar una relación de ese tipo, prefieren no hacerlo.

Por otra parte, Arriagada (2013), en un estudio realizado en la comuna de Santiago llega a conclusiones similares a las planteadas por Luna. Sostiene que se han desarrollado redes clientelares a partir de un encadenamiento vertical de vínculos de media- ción política personalizada, basados en la solución de problemas particulares a cambio de apoyo político. El rol antiguo de intermediación y brokerage vertical de autoridades nacionales y locales como intermediadores que fue descrito por Valenzuela (1977) es reemplazado por la conexión entre la estructura municipal y dirigentes sociales de base (Arriagada 2013).

Siguiendo el planteamiento de Auyero (2002), Arriagada nos explica que el tipo de vínculo establecido entre intermediarios y electorado no se basa simplemente en expectativas de retribución material, sino que existirian expectativas de reconocimiento y prestigio; una fuerte expectativa de reciprocidad y obligaciones mutuas de dar/recibir/devolver; además de la expectativa de recibir beneficios materiales derivadas de esta relación. De acuerdo a la autora, suele negarse el carácter real de este intercambio que aparenta ser desinteresado "a través del autoengaño individual y colectivo. Este autoengaño opera a nivel del habitus, como orientaciones no conscientes de las acciones de los agentes" (Arriagada 2013: 15). Los dirigentes sociales (y no políticos) pasan a desempeñar roles importantes como agentes de transmisión y canalización de demandas y soluciones a problemas particulares. Estos dirigentes (líderes de juntas de vecinos, dirigentes comunitarios, etc.) se convierten en articuladores esenciales en las campañas políticas.

Espinoza (2006), analizando tres centros urbanos en Chile, llega a similares conclusiones al constatar la existencia de redes de intermediación a nivel local que se estructuran a partir de las autoridades locales (alcaldes y concejales) pero que, a diferencia de lo descrito por Valenzuela en los años sesenta, se trataría de estructuras que no están monopolizadas jerárquica y verticalmente por tales autoridades. Más bien se trataría de un núcleo central de agentes y círculos sociales diversos. El núcleo básico "aparece permeable a la iniciativa de los participantes locales, casi con independencia de sus posiciones formales" (P. 4). Así, existiría cierta porosidad del círculo de poder central, aunque la inclusión está mediada por el vínculo que los intermediadores tengan con actores poderosos dentro de la red.

Calvo y Murillo (2012) proponen cambiar el foco de atención y centrarse en el electorado y sus expectativas de retribución. En otros términos, los electores tendrían la capacidad de distinguir la probabilidad de recibir una retribución lo que depende de la proximidad de los electores con activistas de partidos (redes políticas) y del autoposicionamiento ideológico. Comparando el caso de Chile con el de Argentina, los autores concluyen que para el caso chileno existe una mayor expectativa de que determinados bienes serán entregados en la medida en que los partidos más afines a su ideología tengan acceso al poder. En cambio, en Argentina, la expectativa es que la entrega de ciertos bienes se materialicen a través de redes de activistas políticos. 


\section{Selección de casos: INTERMEdiación en TERRITORIOS INDÍGENAS}

Desde el retorno a la democracia, Chile fue gobernado por una misma coalición de centro-izquierda (Concertación) (1990-2010). Ese año conquistó el poder una coalición de derecha (Alianza), que gobernaría hasta marzo de 2014. En las elecciones de 2013 el centro-izquierda presentaría a Michelle Bachelet por segunda vez obteniendo un $46,6 \%$ de los votos en primera vuelta, seguida de la candidata de la Alianza que obtuvo un $25 \%{ }^{1}$. Las elecciones para renovar el Congreso se realizan de modo simultáneo con las Presidenciales de primera vuelta y en cada distrito las listas pueden presentar hasta dos candidatos que compiten en lista abierta para llenar dos asientos por distrito.

Nos interesó estudiar las campañas de candidatos en distritos que involucran a las comunas con los más altos niveles de población indígena y con altos niveles de pobreza. La Araucanía es una región al sur del país caracterizada por un nivel creciente de demandas de organizaciones y comunidades mapuche que reclaman recuperación de tierras, beneficios del Estado y protección de su entorno natural.

Elaboramos un diseño metodológico que complementa técnicas de recolección de datos y formas de análisis cualitativas y cuantitativas. Sin embargo, para el seguimiento de campañas nos focalizamos en dos técnicas de tipo cualitativo: (a) entrevistas semiestructuradas a los candidatos a diputado antes y después de la campaña (6 entrevistas), además de 17 entrevistas a actores clave de la campaña ${ }^{2}$; y (b) la observación, acercándonos desde una perspectiva de etnografía en campaña, donde acompañamos a los candidatos en sus diversas actividades en terreno durante los meses de campaña (septiembre a noviembre de 2013). Este enfoque etnográfico nos permitió realizar una descripción profunda de nuestro objeto de estudio (Geertz 1973) considerando elementos como las relaciones entre candidatos y electores, las tensiones que existen durante la campaña, la configuración interna de las relaciones, los procesos que se repiten permanentemente y las transformaciones que experimentan los actores.

Dadas las limitaciones físicas de investigación, seguimos en terreno las campañas de solo tres candidatos. El primer criterio de selección se basó en la cantidad de reelecciones y el vínculo con la zona. En el primer caso, se trataba de un candidato de la zona y cumplía su séptimo periodo como diputado, esperando que tuviese una red de intermediación muy consolidada (García, RN). En el segundo caso, se trataba de un candidato también de la zona pero que sólo competía por segunda vez (Tuma, PPD). Y el tercer caso se trataba de un candidato que también competía por segunda vez, pero que no era de la zona, por lo que era esperable que tuviese menos redes pre-establecidas (Edwards, RN). Decidimos seleccionar solo casos de candidatos que competían a reelección porque de ese modo podríamos verificar si en su trabajo legislativo habían planteado de algún modo el tema indígena y tenerlo como antecedente a la hora de visualizar su despliegue en campaña.

\section{Caracterización de los distritos}

Los dos distritos de competencia de diputados seleccionados (51 y 52) están ubicados en la región de la Araucanía y son principalmente rurales. Se trata de distritos extensos geográficamente, teniendo por principal actividad económica la agricultura, explotación forestal, seguida por el turismo. Políticamente, ambos distritos muestran una fuerte división del electorado entre la Alianza y la Concertación (hoy llamada Nueva Mayoría).

En las comunas que componen el distrito 51 se advierte una fuerte presencia de la identidad indígena mapuche en instalaciones municipales, iconografía y presencia de tradiciones culturales en diferentes espacios públicos. Varias de las comunas de dicho distrito presentan tasas de población indígena superior al $50 \%$ (65\% en Chol Chol, $60 \%$ en Saavedra y $54,5 \%$ en Freire). La tasa promedio de población indígena en estas comunas es de 47,1\%. En este distrito compitieron y ganaron asientos de diputados por segunda vez los dos representantes que seleccionamos, Joaquín Tuma y José Manuel Edwards. Los votos válidamente emitidos fueron 60.612 (46 $\%$ del padrón), obteniendo Tuma un $36 \%$ mientras Edwards un $32 \%$.

El distrito 52 también es extenso geográficamente y presenta mayores desigualdades pues dentro del distrito se encuentran dos comunas con alta concentración de turismo (Pucón y Villarrica) y otras comunas más distantes, rurales y con alta presencia mapuche. La tasa promedio de población indígena es menor en este distrito (31,6 \%) aunque se advierten algunas comunas que concentran mayor población mapuche (Curarrehue $65 \%$, Pucón $47 \%$, y Villarrica $34,8 \%)$. Presenta la particularidad de contar con dos diputados originarios de la zona, muy conocidos en su distrito y que han permanecido en sus cargos por varios periodos (García con 7 períodos y Meza con 4 períodos). Votaron válidamente 60.576 electores (42 $\%$ del padrón), obteniendo García un 23,7 \% y Meza un $32 \%$.

\section{Caracterización de los candidatos}

Algunos aspectos de las trayectorias de los candidatos son relevantes de considerar:

René Manuel García (63 años) nació en una comuna de su distrito (Cunco) y proviene de una familia vinculada a la región. Desde muy joven estuvo involucrado en la actividad privada agrícola y formó 
parte de organizaciones civiles asociadas al campo. Militante RN, se involucró también en la campaña por el "Sí" a la continuidad del general Pinochet en el plebiscito de 1988. Ha sido diputado consecutivamente desde 1989 por el mismo distrito, obteniendo su mayor porcentaje de votaciones en 1997. A partir de esa elección comenzó a descender, lo que se confirmaría el 2013 al llegar a un 23 \%, aunque le sirvió para reelegirse.

Desde el punto de vista de su gestión legislativa, se trata de un diputado poco visible. Ha participado de comisiones de vivienda y transporte. En relación al tema indígena ha tenido una visión productivista y de protección del patrimonio, promoviendo proyectos de ley asociados al rescate de la lengua indígena y otorgar facilidades para venta de tierras indígenas. Presidió además una comisión investigadora que estudió irregularidades en la entrega de tierras a indígenas en la Corporación Nacional de Desarrollo Indígena.

Joaquín Tuma (72 años) nació en Temuco, militante PPD y proviene de una familia que ha estado involucrada en la región en el mundo de los negocios. Fue presidente de la Cámara de Comercio de la zona. Su hermano, Eugenio, es senador por la región con una vasta experiencia política siendo congresista desde 1994. Disputaba su primera reelección obteniendo una importante alza en votaciones, pasando de 22 a $36,6 \%$ en 2013. En su experiencia como diputado, le preocupaban temas asociados al desarrollo productivo, integrando las comisiones de Economía y Vivienda. Sin embargo, patrocinó dos relevantes mociones, una para promover una reforma constitucional que reconociera a los pueblos indígenas (julio de 2010), y otra para establecer mecanismos de representación indígena a nivel nacional (julio de 2012). Asimismo, había promovido políticas de cupos especiales para estudiantes indígenas para participar en programas de intercambio internacional.
José Manuel Edwards (36 años) nació en Estados Unidos, hijo de padres chilenos, y posteriormente se radicó en Santiago. Es ingeniero de la Universidad Católica. Inició su vida política como dirigente estudiantil en la universidad. Trabajó en el sector privado y luego realizó un postgrado en la Universidad de Harvard. Militante RN, en su corta carrera política como diputado logró un importante incremento en su votación pasando de un $15 \%$ en 2009 a un $32 \%$ en 2013. Sus intereses como diputado se asocian con temas de pobreza, participando de las comisiones de Economía y Desarrollo Social y Pobreza. En su actividad como legislador ha patrocinado proyectos vinculados a permitir venta de terrenos indígenas, y superación de la pobreza. Además, había manifestado una fuerte posición respecto de los conflictos en la zona de la Araucanía, llamando al gobierno a enfrentarlo con mano dura y a los propietarios agrícolas a organizarse para demandar el fin a la violencia.

De este modo, aunque los tres candidatos muestran una preocupación de los temas sociales en relación a la problemática indígena, Tuma había enfatizado más en su trabajo legislativo el reconocimiento y la creación de instituciones que incorporaran a los indígenas en la vida nacional en forma diferenciada. En tanto García y Edwards mostraban un fuerte acento en temas productivistas, superación de la pobreza, y represión por hechos de violencia.

Los candidatos, al momento de definir su estrategia de campaña, suelen tener en cuenta la magnitud de las comunas en términos del número de potenciales electores y el comportamiento electoral de esas comunas. Mientras más competitiva la elección, mayor será el incentivo para recorrer cada una de las comunas en busca de votos porque la elección será estrecha. Pero, si se trata de una elección "asegurada", el candidato no tendrá muchos incentivos de recorrer su distrito y probablemente se concentrará en aquellas comunas que le son más favorables y

Tabla 1.

Caracterización candidatos.

\begin{tabular}{|c|c|c|c|c|}
\hline & Edad & Trayectoria & Gasto* & $\begin{array}{l}\text { Municipios } \\
\text { Favorables }\end{array}$ \\
\hline $\begin{array}{l}\text { JM García } \\
\text { (RN) }\end{array}$ & 63 años & $\begin{array}{l}2009 \text { Diputado } 28,5 \% \\
2005 \text { Diputado 34,3 \% } \\
2001 \text { Diputado 34,3 \% } \\
1997 \text { Diputado 37,3 \% } \\
1993 \text { Diputado 35,9 \% } \\
1989 \text { Diputado 27,3 \% }\end{array}$ & US\$ 62.000 & 3 de 7 \\
\hline $\begin{array}{l}\text { J. Tuma } \\
\text { (PPD) }\end{array}$ & 72 años & 2009 Diputado - 22,2 \% & US\$ 42.000 & 5 de 7 \\
\hline $\begin{array}{l}\text { J. M. Edwards } \\
\text { (RN) }\end{array}$ & 36 años & 2009 Diputado - 15,4 \% & US\$ 87.000 & 1 de 7 \\
\hline
\end{tabular}

Elaboración propia. Resultados electorales y gasto declarado en http://www.servel.cl

* Debemos tener cuidado con las cifras de gasto electoral. Como no existen mecanismos de control del gasto electoral, no podemos afirmar que el gasto declarado es el realmente ejecutado. 
donde obtiene un mayor número de votos. Otra variable a considerar es el signo político de alcaldes y concejales. Ellos suelen cumplir un rol central en la intermediación de demandas sociales desde el municipio hacia el diputado y viceversa. Los candidatos suelen organizar sus campañas verticalmente, a partir de una división territorial por municipios y luego por zonas dentro del municipio. Por lo mismo, para los candidatos es vital contar con el apoyo local.

En nuestro caso, los candidatos del distrito 51 requerían ganar dentro de su lista y acumular aproximadamente unos 20.000 votos para resultar electos. Ninguno de los dos candidatos (Edwards y Tuma) enfrentaban amenazas serias a su reelección. De hecho, en el resultado final ambos más que duplicaron su votación en comparación con sus compañeros de lista. En el caso de Tuma, se advertía un escenario todavía más favorable pues contaba con un marco de votaciones de las municipales muy cercano a la coalición de gobierno. En cinco de las siete comunas de su distrito contaba con el apoyo declarado de los alcaldes. Tuma, para ganar, tenía que concentrarse en la zona de Carahue y Nueva Imperial y fidelizar la votación histórica en el resto de las comunas. Su compañero de lista (Juan Carlos Paillalef) realizó muy poca campaña y la centró en la zona de Saavedra y Carahue donde tenía alguna base de apoyo. Los principales desafíos para Tuma eran Chol Chol y Saavedra, dos comunas dirigidas por alcaldes independientes pro Concertación pero que mantenían cierta distancia con el diputado que corría a reelección por primera vez.

Entre tanto, Edwards enfrentaba un escenario algo más competitivo por dos razones: primero, de las siete comunas del distrito solo una era gobernada por un alcalde de su partido (Freire). Pero adicionalmente, enfrentaba la competencia interna de Mauricio Ojeda, un independiente pro-UDI que era de la región y que enarboló un discurso fuertemente regionalista, criticando con esto al "gringo" Edwards por venir de fuera de la región.

En el caso del distrito 52, García también enfrentaba un escenario competitivo, ya que venía enfrentando una caída en su nivel de votaciones desde el año 1997. Aunque era muy conocido en la zona y contaba con un fuerte apoyo de su partido en comunas clave del distrito (Villarrica, Pucón y Loncoche), su compañero de lista Francisco Reyes (UDI) estaba realizando una millonaria campaña que amenazaba la reelección de García ${ }^{3}$.

\section{Resultados}

Nuestro trabajo de campo confirma lo señalado por la literatura reciente sobre patrones de vínculo clientelar. Los candidatos efectivamente establecen una red de relaciones políticas y sociales que les permiten entrar en contacto con potenciales electo- res. Pero también constatamos innovaciones en las estrategias de campaña que no han sido capturadas por la literatura. Sugerimos que se explicitan dos estrategias de campaña, una tradicional que recoge tácticas de campaña bastante analizadas (redes de intermediación jerárquica, contacto cara a cara, atención personalizada, etc.) y una "profesionalizada" que racionaliza recursos, define prioridades y busca la eficiencia en la distribución de los recursos humanos y financieros de la misma. Ambos tipos de campaña han resultado exitosos electoralmente, aunque anticipamos una tendencia hacia la profesionalización de las mismas, en la medida en que ellas permiten una expansión de los contactos con potenciales electores. A continuación explicitaremos estos dos tipos de campaña encontradas.

\section{Campaña tradicional: la intermediación personalizada}

En nuestros casos, los candidatos Tuma (PPD) y García (RN) representan claramente una estructura tradicional de campaña. Esta estrategia la describió Valenzuela (1977), Luna (2010) y Arriagada (2013) para el caso de Chile, o Auyero (2002) para el caso de Argentina. Se basa en estructuras partidarias preestablecidas que incorporan intensos mecanismos de intermediación clientelar y gestión de recursos públicos con fines electorales. Esta red territorial es jerárquica e incluye, en el caso que analizamos, principalmente a alcaldes y concejales.

Este tipo de campañas no necesariamente depende de la cantidad de años en el ejercicio del poder. De hecho, en nuestro caso observamos una campaña de un candidato que viene siendo reelecto desde 1989 y que ha desarrollado una extensa red de relaciones a nivel territorial, y otro candidato que se presentó por primera vez a una elección el 2009. Lo que parece resultar clave aquí es la trayectoria de los candidatos: se trata de dos personajes con vasta experiencia política en el sector privado, con vínculos con partidos políticos tradicionales y ambos herederos de una tradición política familiar asociada a la participación de organizaciones gremiales y/o políticas.

\footnotetext{
"Yo hice la campaña con muy poca gente. Aproveché el apellido, me moví mucho con los medios locales y con la red que tenía mi hermano... que es lo más importante de todo. Esa red, construida por muchos años, significa que sabemos quién es el presidente de la comunidad, quién es el secretario, dónde viven, dónde están" (candidato a diputado).
}

Los candidatos establecen una estructura de campaña tempranamente en el año electoral con un comando central a cargo de un jefe de gabinete y dos o tres personas de confianza como asistentes. Se establece además una coordinación con alcaldes o, en su defecto, con representantes del concejo municipal del mismo partido en cada territorio. 
El candidato y su equipo definen la agenda de salidas al terreno y ven el día a día de las actividades de campaña. Las visitas son coordinadas entonces con los alcaldes y concejales que asumirán la responsabilidad de planificar reuniones con dirigentes de la comunidad o grupos afines al candidato. Usualmente, las visitas son por un día completo e involucra tres o cuatro reuniones con un promedio de 15-20 personas cada vez.

A una semana de las elecciones, participamos activamente en la campaña de uno de los diputados. Nos invitó a visitar algunas comunidades en su vehículo. Tanto José, el jefe de gabinete del diputado, como Amelia, la encargada de la oficina, van contándole lo que se viene durante la jornada: reunión con comunidad mapuche en Mahuidanche, reunión con comunidad en alto Pitrufquén (Mihuallín bajo). El diputado pide que le dejen libre el sábado antes de la elección. Íbamos camino a una zona rural con lecherías y pequeños agricultores. En el trayecto, José señala que hablaron con Marcos Colichén y que él asegura todos los votos de la comunidad.

Por su parte, Amelia le cuenta al diputado lo que ha pasado en la comuna: la cantidad de cartas que han recibido, los carteles que los contendores han sacado, la gente que ha ido a la oficina a preguntar cómo debían votar, etc. El diputado pregunta si le arreglaron los lentes al lonko Valentín, un lonko líder de la comunidad "de allá arriba" dice, mostrándonos las montañas por la ventana. "Que eso debe estar listo antes del domingo" (día de la elección).

Una mujer representante de la comunidad le organizó la reunión con unas 15 personas. Al llegar, el diputado saludó a cada uno de los presentes, se sentó y preguntó directamente qué tipo de problemas tenía la comunidad. Preguntaron sobre el programa social "Chile indígena". La comunidad estaba ilusionada con obtener un tractor a través de aquel programa. El asesor del diputado explicó sus características indicando que el actual gobierno había dejado muy pocos recursos y para atender sólo dos localidades en la Araucanía. El diputado no conocía de su implementación aunque critica al gobierno por crear este tipo de instrumentos con pocos recursos. De igual modo, les aconseja que sigan el proceso del programa si los invitan. La relación del diputado con los asistentes es cordial. Luego, ya finalizando la reunión, se les muestra un facsímil de voto y se explica cómo será el día de la elección. Esta instrucción va repitiéndose en cada reunión a que asistimos. Además, los asesores del diputado hablan con los participantes sobre la forma en que se organizará el proceso de concurrencia a votar, asegurándoles transporte que será provisto por el comando del diputado.

Los diputados cuentan con algunas oficinas comunales fijas y con otras que solo funcionan en época de campaña. Algunos colaboradores trabajan por campaña (pagados o voluntarios) y otros trabajan formalmente con el diputado durante el año. Los permanentes son el encargado de oficina comunal y el jefe de gabinete. Este último es quien se encarga de vincular los diferentes trabajos territoriales, generando en conjunto la agenda definitiva que debe seguir el diputado durante su semana. El resultado es la organización de actividades que debe llevar a cabo en: Valparaíso (sede del Congreso), Temuco (ciudad capital regional), Oficinas Comunales y también, la sede del Congreso en Santiago (para vincularse con ministerios).

A esta red territorial se suma el trabajo rutinario que desarrolla durante el año la oficina parlamentaria de cada diputado. Algunos diputados tienen una oficina central en el distrito, mientras otros han abierto dos o tres pequeñas (con una persona remunerada a cargo) en algunas comunas relevantes para el candidato. En la oficina distrital suele trabajar una secretaria durante todo el año y un abogado que atiende consultas y peticiones de las personas. Lo anterior le permite al candidato que va a la reelección contar con una red pre-establecida de contactos y vínculos directos con distintas personas y comunidades (teléfonos y nombres de contacto).

La principal función de las oficinas parlamentarias es la asesoría a la ciudadanía sobre trámites institucionales y la canalización de demandas y/o denuncias ciudadanas o personales de los habitantes de cada comuna. Esta situación se replica en los comandos de cada uno de los candidatos y supera los tiempos de campaña, ya que es un trabajo que se da día a día, pero que en campaña se intensifica. Cada oficina tiene un encargado que por lo general está ligado al partido del candidato y conoce el funcionamiento político de la comuna. Así explica una encargada de local su involucramiento con esta actividad:

\begin{abstract}
"Me integré al PPD el año 88 [...] Pasaron por la casa, andaba un grupo de gente y, a pesar de que mi mamá me decía "no, no, política no", me inscribí sin pensarlo mucho... Empecé a participar del partido, acá en Pitrufquén. Hace unos 4 años atrás fui la presidenta del partido, después me reeligieron, termino mi período el próximo año [...] Yo trabajaba en un colegio, y un día me llaman y me dicen que me venga a trabajar con ellos a la oficina..."
\end{abstract}

Y en relación a sus funciones, sostiene:

"Oriento a la gente en trámites, trámites que no costaba mucho hacerlos, pero la gente en los municipios no tiene la voluntad [...], ayudar en regularización de vivienda, de cambio de funcionarios públicos, gendarmería, que llegan a la oficina parlamentaria a pedirle ayuda a los parlamentarios para que los trasladen. [...] Llegaban muchísimas peticiones, yo creo que en el período de campaña aumentaron como un $200 \%$. Era mucho, mucho, porque en tiempo de campaña empiezan a hacer bingos, beneficios, empiezan a juntar plata para las giras de estudios, en fin... son cartas todos los días." (Encargada de oficina en Pitrufquén). 
Finalmente, la estructura de campaña se nutre también de una red de militantes de partido que forman parte de reparticiones del estado a nivel regional que apoyan indirectamente al candidato ya sea en algunas actividades en terreno o bien a través de la resolución de problemas específicos como veremos más adelante. Este año se sumaron por primera vez los candidatos a Consejeros Regionales (CORES) que se integran a la red de trabajo del candidato. Una nueva ley estableció la elección directa de CORES, lo que implicó que ellos realizaran campaña asociada con los candidatos a diputado.

Desde el punto de vista del despliegue de la campaña, se realiza a partir -principalmente- de reuniones cara a cara con potenciales electores. Estas reuniones se organizan en centros comunitarios o en casas particulares de dirigentes ya sea de juntas de vecinos o comunidades indígenas. En estas reuniones el candidato suele estar acompañado por su jefe de gabinete, un concejal o alcalde, y el o la candidata a CORE que está apoyando. El objetivo principal de las reuniones es escuchar las demandas específicas de los asistentes y buscar la solución a estos petitorios.

De manera paralela, el diputado y sus funcionarios son quienes -en algunas ocasiones- realizan la labor de "intermediarios" entre la ciudadanía y los servicios públicos con la finalidad de agilizar los procesos y obtener una buena respuesta para las demandas de los ciudadanos.

Adicionalmente, pudimos observar la realización de actividades sociales en vinculación con alguna organización. En los últimos años ha tomado mucha fuerza la realización de "bingos" donde el candidato obsequia los premios que quedarán a disposición de quienes participan y ganan en juegos de azar. Hasta muy recientemente los candidatos solían también organizar asados, aportando vaquillas y vino en actividades sociales que congregaban a mucha gente. Esta práctica fue menos común en la última elección seguramente porque se ha instalado en el discurso como una mala práctica de clientelismo con sectores pobres.

Otra actividad es la participación en eventos públicos que suelen estar organizados por el municipio (inauguraciones de centros u obras, actos oficiales), y donde se aprovecha la oportunidad de intervenir y/o intercambiar con potenciales electores. Finalmente, el candidato participa en eventos -mucho más esporádicos-donde concurre el o la candidata presidencial. Esta actividad suele ocurrir en la capital regional (en nuestro caso, Temuco), por lo que el candidato apoya con recursos para que sus propios electores se trasladen hacia ese evento pagando microbuses para la ocasión.

Desde el punto de vista del despliegue de la campaña, observamos un amplio dispositivo de meca- nismos de intercambio material y simbólico con los electores. Aunque algunos estudios han puesto mucho énfasis en la entrega de obsequios simbólicos de campaña (lentes de sol, calendarios, espejos, lápices, etc.), en realidad el despliegue de la campaña va acompañado de un conjunto mucho más relevante de intercambios en que convendría poner atención. Los organizaremos en tres: información, apoyo material, e influencia.

Información. El candidato en su despliegue territorial cumple una labor importante de informar a los electores de una amplia gama de asuntos legales y de políticas públicas. En las reuniones con sus electores, el candidato responde a dudas sobre acceso a beneficios del estado, a dudas legales, información de nuevos programas sociales gubernamentales, etc. Cuando se trata de asuntos comunitarios de mayor envergadura, usualmente el jefe de gabinete recoge dudas de los asistentes y promete una respuesta a dichas interrogantes en el futuro.

Por ejemplo, participamos en una reunión en la localidad de Cunco. El candidato llega y saluda, regala lápices, calendarios y votos. Todo en un tono alegre y de confianza. Conoce a la gente por su nombre de pila. Bromea con las mujeres y hablan de su familia. Se acomodan las sillas en círculo, nos sentamos todos. “¿Algún tema?”, pregunta. Uno de los asistentes pregunta por casetas del Serviu (Servicio de Vivienda y Urbanismo). Respuesta: el proyecto está postulado, la municipalidad postuló el proyecto... Otra persona pregunta cómo funciona lo de cambiar de casas viejas por nuevas. Se le explica que se necesita 1 millón de ahorro para favorecer a gente que tiene más ahorro. El mismo diputado toma su teléfono celular y llama al Serviu para saber cómo va el trámite de la persona ahí presente. Habla fuerte, le da nombre, número de identificación y fecha en que postuló y averigua en qué va la postulación. Al otro lado del teléfono buscan el caso y le explican las etapas de la postulación... Pedro, el jefe de gabinete, registra todo.

Apoyo material. El candidato también invierte recursos materiales durante su campaña, lo que se traduce en compra de obsequios para bingos, regalos de implementación deportiva para clubes de fútbol, apoyo con recursos para la realización de obras en centros comunitarios, entrega de dinero para giras de estudio, pago de traslados para actividades de organizaciones, operativos de salud y entrega de dinero en efectivo en bajas sumas a personas que presentan problemas. Los candidatos tienden a evitar la particularización en la entrega de recursos, optando más bien por apoyar iniciativas colectivas de modo que pueda multiplicar su apoyo. Otra dimensión de este apoyo material es el pago de microbuses para trasladar a personas el día de la elección y que se concreta, ya sea mediante el pago del combustible para algunos dirigentes que trasladan gente a votar, o, directamente, la contratación de buses. 
Nadie reconoce abiertamente la entrega de regalos durante la campaña. Los dos candidatos que seguimos, que responden a este modo tradicional de hacer campaña, aminoran la importancia de la entrega de regalos: uno regala a las mujeres asistentes "espejos mágicos" con su nombre impreso, bromeando con que si se miraban en ese espejo se verían 5 años más jóvenes. El otro repartía unos papeles de unos $15 \times 20 \mathrm{~cm}$ : por un lado era una bandera chilena y por el otro una foto del candidato.

Influencia. Otra forma de intercambio es la oferta de su influencia para la solución de problemas individuales o colectivos. En muchas de las reuniones se produce un primer momento colectivo, donde el candidato ofrece soluciones a problemas específicos de la comunidad como ripiado de caminos, estudio de caudal de aguas, influencia para la materialización de mejoras en sedes sociales, mejoras en consultorios de salud, solución a problemas en escuelas. Allí el candidato ofrece sus oficios para interceder en la solución de los problemas con las autoridades de la alcaldía, el Gobierno regional o incluso el Gobierno central. Pero además, se producen también muchas reuniones privadas en las que el candidato recibe solicitudes concretas y que son formalizadas en una carta o simplemente de palabra.

El rol de intermediario entre el nivel local y uno regional y central es clave dentro de la campaña. Aquello es explicitado por todos los candidatos:

\begin{abstract}
“¿Cómo se consiguen las cosas? Primero parto por el SEREMI, voy donde el SEREMI de obras públicas, llevo una foto y le digo 'mira el puente, está que se cae, hagamos esto'. Funciono con los consejeros regionales, tenemos mayoría en los consejeros regionales y les digo que aprieten al intendente, que necesitamos esto [...] Voy donde el senador y le digo 'no tengo mayoría aquí en la comisión pero tú apriétame al ministro'” (Candidato a diputado).
\end{abstract}

Las campañas políticas enfatizan muy poco los aspectos programáticos e ideológicos. El discurso de campaña se basa fuertemente en destacar los aspectos personales del candidato (confianza, experiencia, influencia) y muy poco en aspectos asociados a los temas programáticos. Las características sociodemográficas de los distritos que consideramos implicarían que, teóricamente, los candidatos debieran poner atención a temas vinculados a la problemática indígena, incluyendo cuestiones relativas a reconocimiento, restitución de tierras, e impacto de la implementación del Convenio $169^{4}$ de la OIT.

Durante la campaña, los candidatos muchas veces se reunieron con comunidades indígenas. Sin embargo, en tales reuniones muy pocas veces se abordó específicamente el tema indígena. Y esto por dos razones. Primero, los candidatos suelen iniciar sus actividades escuchando las demandas de la gente y muchas veces estas demandas no aluden a problemas de reconocimiento indígena, sino más bien de acceso a beneficios del estado o solución de problemas materiales que aquejan a individuos o la comunidad como planteábamos más arriba. En segundo lugar, los candidatos (de ambas coaliciones) tienden a compartir una visión "productivista" de las relaciones entre el pueblo mapuche y el estado de Chile. Esto quiere decir que los candidatos enfatizan la necesidad de que los indígenas adquieran habilidades y conocimientos para integrarse al modelo productivo nacional. En palabras de un candidato:

\footnotetext{
"Te voy a decir lo siguiente: yo creo que Chile tiene un tren de la victoria con 14 carros, que son todas las regiones que exportan (cobre, vino, fruta, salmones). Nosotros no estamos ahí. Yo necesito agregar el carro 15, que es la región de la Araucanía [...]. Para ello requerimos políticas públicas que se dirijan a ello para acompañar especialmente a la mayoría de las comunidades mapuche a vincularse con el mercado...." (Candidato a diputado).
}

Retóricamente, existe alguna diferencia entre la Nueva Mayoría y Alianza en relación a esta temática. El candidato de la Nueva Mayoría se refiere en las reuniones en términos bastante generales a las propuestas programáticas de la coalición en materia indígena (creación de un ministerio de asuntos indígenas por ejemplo) y en el fortalecimiento de la representatividad indígena en el sistema político. Pero también destaca públicamente las dificultades de las propias comunidades para organizarse colectivamente para demandar sus derechos. Asuntos específicos relacionados con CONADI o con el Convenio 169 no forman parte del discurso de este candidato. A ello se suma la percepción del candidato sobre las dificultades para plantear algunos de los temas asociados a derechos indígenas:

\begin{abstract}
"Yo terminando una reunión, de casi dos horas, pregunté 'quiénes de ustedes sabian lo que significaba la consulta del 169'. ¿Sabes cuántos me contestaron que sabian? Dos de cuarenta y ocho [...] Entonces, mientras ellos no conozcan esto... Estamos hablando de personas que no tienen mucha educación, que no tienen mucho conocimiento, y que hay que informar con peras y manzanas [...]. (Candidato a diputado).
\end{abstract}

Las campañas electorales muy pocas veces sirven para socializar ideas o debatir programas. Los candidatos suelen enfatizar, en primer lugar, su capacidad para resolver problemas o interceder frente a otras autoridades del gobierno regional o central en la solución de conflictos que aquejan a las comunidades. En segundo lugar, colocan un fuerte énfasis en visiones que tienen relación con la integración de los indígenas al modelo productivo nacional. Finalmente, y en el caso de la derecha, se enfatiza la necesidad de que las autoridades actúen con firmeza frente a las expresiones de violencia producto de demandas de algunos grupos mapuche. En opinión del candidato de la Alianza: 
"En mi distrito, felizmente, los mapuche están mucho más integrados, están viviendo del turismo, están haciendo otras cosas, y se incentivaron a tener etnoturismo, vender su artesanía, a que el primer cordero del año no se come en octubre porque ese cordero costaba 20.000, ahora lo venden en enero y febrero y vale 50.000. O sea, han adaptado su productividad... lo que ha sido muy bueno...". (Candidato a diputado).

\section{Campaña profesionalizada: la intermediación despersonalizada}

Siguiendo a otro candidato de la IX región, vimos que la intermediación entre representantes y electores ha encontrado una nueva expresión en el surgimiento de lo que denominaremos "campañas profesionalizadas". Este candidato tiene las características de ser joven y con solo un período de diputado en sus espaldas.

El jefe de campaña nos explica que el diputado tiene un equipo de unas 7-8 personas que forman el comando: un abogado, un jefe de campaña, un profesional audiovisual, una fotógrafa, y tres choferes. Y además contratan a voluntarios para el "puerta a puerta". Para la campaña trabajaban con doce radios y para decidir con cuáles trabajar hicieron un estudio de audiencia. Pagaban avisos en dos radios comunitarias y en radio Bío-Bío ${ }^{5}$, siendo esto muy costoso, sobre todo en tiempo de campaña: "en tiempo normal me costaba $\$ 3.800$ el minuto, ahora $\$ 7.000$ [US\$12]. También se mandó a hacer merchandising a China, los carteles, bolsas, cámaras Polaroid, ahorrando en eso "como \$10 millones" [US\$16.000]".

Según ellos, hacen una diferencia respecto de los otros candidatos en el puerta a puerta: "Va el candidato y seis personas. Van en zigzag, así avanzan y es más eficiente. El diputado pasa, saluda y entrega algún regalito y los demás van un poco más adelante preparando las casas para que esté la gente en la puerta esperando al candidato. Siempre va el camión del comando con música y una persona disfrazada del candidato". Realizan además un despliegue simultáneo de dos camionetas con propaganda y parlantes con el objetivo de dar la impresión de que el candidato está presente en todo el distrito.

Al preguntarles por si organizaban reuniones cerradas con electores como sucedía en la campaña tradicional, nos dicen que en tiempos de campaña preferían evitar las reuniones con poca gente porque estas siempre venían con peticiones detrás. Se enfocan en mucho trabajo de terreno, en una campaña más lúdica, con música, fotos con la familia, entrega de obsequios simbólicos como flores a las mujeres. En la medida en que avanza la campaña se van aumentando los avisos en la radio, carteles en las calles y plazas. Adicionalmente, tienen una estrategia de difusión en las redes sociales y mensajes de texto.
"Vemos que el Fan page tiene 180 personas nuevas a la semana. Una adherencia de 71.000 personas. Esto lo tenemos clasificado por comuna y rango etario. También enviamos mensajes masivos, para lo que se creó una aplicación web. Después se georeferenciará y tendremos mucha más y mejor información sobre el distrito." (Jefe de campaña).

Desde la perspectiva de la estructura de campaña, esta se basa en un equipo contratado y cercano al candidato que se constituye como un comando central. Sin embargo, a diferencia de una campaña tradicional, donde se trata de personas que mantienen un lazo de confianza y lealtad con el candidato y que pueden asumir distintos roles dentro de la campaña, aquí se observa una definición de roles más clara (jefe de campaña, encargado de comunicaciones, relación territorial, vínculo legal). En el caso de Edwards, el jefe de campaña era un joven profesional que había colaborado en otras campañas de la Alianza (UDI y RN), y que por lo tanto había desarrollado un expertise en el trabajo territorial.

Aunque el candidato establece vínculos con dirigentes locales de comunas (alcaldes y concejales), su relación es menos estrecha que en el caso que observábamos antes. El candidato tiende a trabajar con algunos actores de confianza del partido en las comunas, pero radica especialmente su atención en el trabajo anterior realizado por su oficina parlamentaria.

En este sentido, el despliegue de la campaña es diferente a lo que describíamos para el caso de la campaña tradicional. Primero, el candidato y su equipo realizan una planificación de su estrategia de campaña a partir de la identificación de los territorios donde es más probable obtener votos y donde resulta más eficiente obtenerlos. Así, los principales esfuerzos se dedican a recorrer en un sistema de "puerta a puerta en zigzag" los pueblos de comunas específicas. El gasto de recursos y tiempo del candidato trasladándose a zonas apartadas del distrito es considerado como ineficiente, por lo que se busca en la campaña tener el mayor número de contactos directos con personas, y aquello ocurre en los pueblos.

En segundo lugar, tal cual ocurre con las campañas tradicionales, la oficina parlamentaria del candidato atiende consultas y requerimientos durante el año. Ahora bien, estos datos son recogidos y sistematizados de modo que pueda establecer una base de datos de contactos que le sirven al candidato para mantener un vínculo con los electores ya sea a nivel telefónico (vía mensajes de texto) o mediante contactos directos durante la campaña. Además, los encargados de la oficina también sistematizan el tipo de consultas y peticiones que llegan a la oficina, de modo que puedan identificar las prioridades de las personas y organizar la estrategia de campaña de acuerdo a dichas prioridades.

El despliegue de la campaña, entonces, es muy distinto a lo que observábamos en el primer caso. 
Aquí vemos un verdadero "barrido" del distrito, focalizado en pueblos y ciudades. Cercanos al candidato nos dijeron que además de la sensación de "alta presencia", se realizaron con anterioridad a la campaña algunos estudios para conocer la percepción del electorado sobre el candidato y así poder focalizar la campaña en ciertos aspectos específicos que hayan resultado los puntos fuertes según los electores.

Otra estrategia fuerte de este tipo de campaña más profesionalizada es el modo en que se destinan los recursos. Más que gastar el dinero en reuniones, asados, eventos, ellos prefieren realzar la idea de "estar permanentemente en terreno", lo que hacen apareciendo mucho en la radio, sobretodo en tiempo de campaña.

Se suma a esta estrategia la realización de "operativos médicos" en los pueblos visitados por el candidato, en los que dos o tres médicos ofrecen atención gratuita a las personas. Los médicos entregan recetas y/o muestras de remedios para las personas que visitan el lugar (que generalmente es el comando del mismo candidato).

Llegamos a una sala de reuniones en la localidad de Pitrufquén, donde se tenía preparado un operativo médico. Unas 25 personas que escucharon el día anterior en la radio el llamado a registrarse esperaban la llegada de los doctores. Una mujer que esperaba ser atendida, Beatriz, nos cuenta que los candidatos hacen este tipo de cosas y que se ganan los votos y después cuando son elegidos no vuelven a dar las gracias. Nos cuenta que son todos iguales. Le preguntamos si votaría por Bachelet y dudó, pero a los 5 minutos dice que ella siempre ha votado por la Democracia Cristiana (un partido de centro): "pero que hay que aprovechar todo lo que se les ofrece".

La profesionalización de la campaña se hace evidente en materia de propaganda. Se trata de una campaña con alta visibilidad y calidad técnica en términos de carteles y avisos de radio. Lo anterior se planifica con anticipación mediante la confección de propaganda en China, lo que reduce su costo. Asimismo, los encargados de la campaña realizan un estudio de fortalezas y debilidades del candidato para poder definirlo con un concepto. En esta oportunidad buscaron "humanizar" al candidato a través de una campaña emotiva que lo mostraba como padre y esposo, muy cercano a su familia. En los carteles aparecía el candidato junto a su hijo recién nacido y su señora, junto al lema "mucho más Rojo". Se trataba de un juego de palabras contraintuitivo, pues aludía al hecho de tener el cabello rojizo y recién haber nacido un hijo muy parecido a él. Sin embargo, también implicaba una alusión irónica hacia la izquierda por tratarse de un candidato de derecha. Tal como advertíamos al inicio de este trabajo, la candidatura de Edwards fue una de las más costosas de las campañas que se presentaron en los distritos 51 y 52 y aquello se hacía evidente en su despliegue comunicacional.
"Durante febrero tuve la oportunidad de acompañarlo a él y la familia por el distrito [...]. Una de las cosas que me tocó ver es cómo reaccionaba la gente con la guagua de Manuel, que es su hijo, un diputado chico, una réplica de él, y era impresionante... Y la gente hablaba muy bien de que anduviera con la familia [...] y me convencí de que eso era un eje súper importante en la campaña, no mostrar solo a la persona, sino también a la familia. Y de ahí nacieron varias cosas importantes, nació la imagen de campaña ahí y nació un tema que se pulió con el tiempo, que era el "mucho más rojo"." (Jefe de campaña).

Aunque se trata de una campaña más despersonalizada, no es que no existan mecanismos de intercambio entre el candidato y los electores. También se dan estos, pero una de las características de este estilo de campaña es que están más intermediados por la estructura de campaña. Como se dan menos oportunidades para reuniones con organizaciones (que suelen estructurar mejor sus peticiones), los intercambios en tiempos de campaña que suelen prevalecer son dos: a) información para acceder a beneficios del Estado $y, b$ ) influencia a través de los agentes de la oficina parlamentaria para interceder ante instituciones del Estado y resolver problemas individuales o colectivos. El apoyo material se da principalmente por la vía de operativos médicos, entrega de remedios, y apoyo para algunas organizaciones en la realización de bingos.

Lo anterior no significa que el candidato no sostenga y nutra sus relaciones con comunidades de la zona. De hecho, durante el tiempo no electoral el candidato reconoce que asiste a reuniones con comunidades más alejadas y en donde se le plantean inquietudes, demandas y peticiones que son procesadas por el equipo de trabajo estable del diputado en el distrito. Desde el punto de vista discursivo, los principales énfasis de la campaña fueron el tema del acceso a la salud, el fortalecimiento de la familia, y el mejoramiento de los ingresos.

Los aspectos asociados a reconocer derechos indígenas no forman parte de su campaña. Su énfasis estaba puesto en el desarrollo de políticas sociales y productivas para mejorar las condiciones de vida social en una de las regiones más pobres del país. Al ser entrevistado, el candidato valora discursivamente la importancia de la identidad cultural indígena pero también reconoce que no forma parte de su campaña y que el tema es más complejo que simplemente la reparación por la vía de la devolución de tierras o por el reconocimiento constitucional.

En síntesis, la campaña profesionalizada es racional, estructurada, eficiente. Requiere de mucha planificación y estudio de proyección de votos, para así focalizarse en cierto perfil de electores. Es una campaña sin grandes discursos, y la gestión de las demandas de la comunidad se realiza a través de las personas que trabajan con el candidato en regiones durante el año (en particular el abogado). 
Finalmente, la imagen de la campaña resulta fundamental en la estrategia para atraer votos, repartiendo mucho merchandising y afiches publicitarios de muy buena calidad (el estilo gringo en esta campaña era la marca del candidato).

\section{Discusión}

Confirmando lo señalado por la literatura reciente, vemos que el vínculo candidatos-electores se basa fuertemente en relaciones de intermediación esencialmente no programáticas. La forma en que se estructura una campaña, su despliegue temporal y el tipo de discurso de los candidatos tiene una lógica que acentúa sus atributos individuales; que destaca la capacidad de resolver problemas cotidianos de la gente; y que coloca énfasis en el bienestar inmediato de los electores.

No obstante, encontramos diferencias en las modalidades de interpelar al electorado. Identificamos dos tipos de campaña (tradicional y profesionalizada) que se proyectan como dos modalidades de intermediación frente a los electores. ¿Podemos extender este argumento al resto del país? Como vimos, ya varios autores han sugerido el predominio de vínculos no programáticos a la hora de obtener votos (Luna 2010; Contreras 2012; Arriagada 2013). Lo que aquí se sugiere es que dicho objetivo se logra por estrategias diferenciadas que parecen depender más de la trayectoria del candidato que de su posición política o permanencia en el mismo distrito. También advertimos la total ausencia de temas programáticos significativos a nivel discursivo en las candidaturas. Con mayores o menores matices, la cuestión indígena, que es relevante en el discurso nacional y en la presentación de proyectos, suele quedar relegada en las campañas a un discurso inicial que valora la cultura y tradiciones. Sin embargo, la atención de los candidatos suele radicarse en la solución de problemas particulares de sus electores.

Desde el punto de vista teórico, nuestra investigación aporta a la reflexión sobre la configuración de patrones de intermediación entre partidos y ciudadanía. Por tratarse de un estudio realizado en una zona geográficamente distante de grandes urbes, nuestros resultados reflejan una doble aproximación. Por una parte, comprueba la vigencia de lo señalado varias décadas atrás por Valenzuela en political brokers, en la que el diputado ejerce un rol interme- diador de demandas locales con otros actores de la esfera estatal, en tanto los alcaldes y concejales se transforman en mediadores del acceso a los territorios. El éxito electoral de este broker dependerá de su capacidad para sostener sus redes de intermediación a nivel local y de movilizar los recursos de su partido para alcanzar aquello en su distrito. Varios incentivos institucionales podrían explicar esta tendencia: el hecho de contar con un sistema electoral de lista abierta con voto nominal que personaliza la campaña, la baja raigambre territorial de los partidos y la poca relevancia del financiamiento estatal de campañas. Lo anterior lleva a que el éxito dependerá del carisma del candidato, de su propia capacidad de desarrollar una red y de su capacidad de recaudación de recursos para sostener su campaña.

Pero también advierte simultáneamente de la emergencia de nuevas tendencias en el desarrollo de campañas. En la medida en que se debilitan las redes partidarias y se advierte fragmentación social, los candidatos optan por estrategias propias basadas en la conformación de sus propias redes de intermediación y con una fuerte inversión en publicidad. La profesionalización de las campañas busca personalizar y desideologizar la acción política.

En ambas vertientes (tradicional o profesional) nos enfrentamos a una relación representante-representado que se sustenta en el carisma específico del candidato, en la resolución específica de problemas y en prácticas y rituales políticos particularistas más que colectivos. Las campañas pasan a depender del tipo de vínculo emocional que se establece. La "venta de soluciones" se transforma en la vía para acceder y mantenerse en el poder.

\section{Agradecimientos}

Ambos autores son profesores de la Universidad Diego Portales e investigadores asociados del Centro Interdisciplinario de Estudios Interculturales e Indígenas -FONDAP-. Claudio Fuentes es, además, investigador senior del Proyecto Núcleo Milenio NS 130008. Maite de Cea es investigadora responsable del proyecto FONDECYT No11140083 sobre descentralización cultural y construcción de identidad territorial, y el artículo se escribió en el marco de los proyectos señalados. Los autores agradecen los comentarios de los revisores anónimos y de los editores de la revista.

\section{Notas}

Finalmente, Bachelet obtendría un $62 \%$ de los votos en segunda vuelta, mientras la candidata Matthei de la Alianza un $37 \%$.

2

Justificamos la elección de las entrevistas semiestructuradas como fuentes de recolección de datos puesto que nos permiten identificar, no solo conexiones lógicas, sino emocionales que se articulan en un mismo discurso sobre ciertas experiencias y hechos. Y por su parte, la etnografía en campaña complementó la información recolectada en las entrevistas eficazmente, dado que así podíamos ver en el terreno las prácticas de los actores, tanto de los representantes como de los representados y observar cómo funcionaba el vínculo entre ellos en un período de campaña electoral. 
En las cuentas del Servel, mientras García declaró haber gastado 62.000 US\$, Reyes declaró 98.000 US\$ (ver http://www.servel.cl).

En el año 2008 el Congreso Nacional aprobó la suscripción del Convenio 169 de la Organización Internacional del Trabajo que establece la obligatorie-

\section{Referencias Bibliográficas}

Altman, D. 2006. "Reformas institucionales para el mejoramiento de la calidad de la democracia en Chile". Pp. 49-86 en Desafíos Democráticos, editado por C. Fuentes et al. Santiago: LOM.

Arriagada, E. 2013. "Clientelismo político y participación local. El rol de los dirigentes sociales en la articulación entre autoridades y ciudadanos en Santiago de Chile". Polis 36:1-21. http://dx.doi.org/10.4067/s071865682013000300002

Auyero, J. 2002. "Clientelismo político en Argentina: doble vida y negación colectiva". Perfiles Latinoamericanos 20:33-52.

Barozet, E. 2003. "Movilización de recursos y redes sociales en los neopopulismos: hipótesis de trabajo para el caso chileno". Revista de Ciencia Política XXIII: 39-54. http://dx.doi.org/10.4067/s0718-090x2003000100004

Calvo, E. y Murillo, V. 2012. "When parties Meet Voters: Assessing Political Linkages Through Partisan Networks and Distributive Expectations in Argentina and Chile". Comparative Political Studies XX: 1-32.

Contreras, G. 2012. "Redes de intermediación política en Chile: retomando la discusión sobre los brokers políticos en el Chile post autoritario". Presentado en el $X$ Congreso Chileno de Ciencia Política, Santiago, 17-19 de octubre.

Díaz, D. et al. 2006. "El secreto de mi éxito. Seis caminos para llegar y permanecer en Valparaíso". Revista de Ciencia Política 26:169-190.

Durston, J. 2009. "Clientelismo político y actores populares en tres regiones de Chile". Universidad de Los Lagos, Chile. Estudio inédito, disponible en http://www.innovacionciudadana.cl/portal/imagen/File/durston $\% 20$ clientelismo\%20regional\%201209.pdf

Espinoza, V. 2006. "Los nuevos agentes políticos locales: revisión de la tesis de Arturo Valenzuela ¿Cómo se articulan el nivel de representación local con el nacional en la arena local y qué papel juegan el gobierno central y las políticas públicas?" Revista Mad 14:1-8.

Fuentes, C. et al. 2006. Desafíos Democráticos. Santiago: LOM.

MAITE DE CEA es socióloga de la Pontificia Universidad Católica de Chile y Doctora en Ciencia Política de la Universidad de Grenoble, Francia. Académica de la Escuela de Sociología y directora del Instituto de Investigación en Ciencias Sociales (ICSO) de la Universidad Diego Portales. Su investigación se ha centrado en la evolución institucional de la política cultural en Chile y en el análisis de los derechos indígenas y su reconocimiento político, legal y cultural en Chile. Entre sus publicaciones están Genèse d'une institution publique pour la culture au Chili: Le Conseil national de la culture et les arts. La Documentation Française, 2011; La omisión a la diferencia. Elites, discriminación y reconocimiento de pueblos originarios en Chile. Ediciones UDP, 2012. dad del Estado de Chile de consultar a los pueblos originarios sobre la implementación de políticas que les afecten directamente.

5 Radio Bío-Bío es una reconocida radio originada en la ciudad de Concepción, en el sur de Chile, pero que tiene alcance nacional.

Geertz, C. 1973. "Thick Description: Toward an Interpretive Theory of Culture". Pp. 3-30 en The Interpretation of Cultures: Selected Essays, editado por C. Geertz. New York: Basic Books.

Levitsky, S. y Murillo, V. 2005. Argentine Democracy: the politics of Institutional Weakness. Penn State: Pennsylvania State University Press.

Luna, J. P. 2010. "Segmented Party-voter Linkages in Latin America: The case of the UDI". Journal of Latin American Studies 42:325-356. http://dx.doi.org/10.1017/S0022216X10000465

Luna, J. P. y Rosenblatt, F. 2012. “¿Notas para una autopsia? Los partidos políticos en el Chile actual”. Documento de trabajo CEP Chile-Cieplan.

Mainwaring, S. y Scully, T. 1995. Building democratic Institutions. Party Systems in Latin America. Stanford: Stanford University Press.

Rehren, A. 1996. "Corrupción y política local en Chile". Revista de Ciencia Política 18:141-153.

Siavelis, P. 2000. "Continuidad y cambio en el sistema partidista chileno: sobre los efectos de transformación de una reforma electoral". Revista de Ciencia Política 20:82101.

Schroter, B. 2010. "Clientelismo politico: ¿Existe el fantasma y cómo se viste?" Revista Mexicana de Sociología 72:141-175.

Szwarcberg, M. 2013. "The microfoundations of political clientelism. Lessons from the Argentine Case". Latin American Research Review 48:32-54. http://dx.doi. org/10.1353/lar.2013.0024

Tironi, E. y Agüero, F. 1999. “¿Sobrevivirá el nuevo paisaje político chileno?". Estudios Públicos 74:151-168.

Valenzuela, A. 1977. Political Brokers in Chile: Local government in a Centralized Polity. Durham: Duke University Press.

Valenzuela, S. 1995. "Orígenes y transformación del sistema de partidos en Chile". Estudios Públicos 58:5-80.

CLAUDIO FUENTES SAAVEDRA es licenciado en Historia de la Pontificia Universidad Católica de Chile (1991) y Doctor en ciencia política de la Universidad de Carolina del Norte (Chapel Hill, 2003). Es profesor titular y director de la Escuela de Ciencia Política de la Universidad Diego Portales. Sus intereses académicos se han volcado al estudio de los procesos políticos en Chile y América Latina. Entre sus obras más recientes se cuenta El Pacto (Ediciones UDP, 2012), El Fraude (Hueders 2013) y el libro co-editado con Alfredo Joignant, La Solución Constitucional (Catalonia, 2015). 